\section{Autoantikörper gegen Mitose-assoziierte Antigene}

\section{W. Stöcker}

Euroimmun Medizinische Labordiagnostika AG, Lübeck,

Deutschland

Englischer Begriff autoantibodies to mitosis-associated antigens

Definition Autoantikörper gegen Strukturen, die vorwiegend in der Mitosephase des Zellzyklus vorkommen oder ihre Funktion ausüben. $\mathrm{Zu}$ diesen antigenen Strukturen zählen:
- MSA-1 (Mitosespindelapparat, Antigen 1, - Autoantikörper gegen Spindelapparat [unzweckmäßige Bezeichnung: $\mathrm{NuMa}])$

- MSA-2 (Mitosespindelapparat, Antigen 2, > Autoantikörper gegen Spindelapparat [HsEg5])

- Midbody ( $\triangleright$ Autoantikörper gegen Midbody)

- CENP-F ( $\triangleright$ Autoantikörper gegen CENP-F)

- Zentriolen ( $\triangleright$ Autoantikörper gegen Zentriolen/Zentrosomen)

- Zentromere ( $\triangleright$ Autoantikörper gegen Zentromere) 